\title{
A Liposome-Based Adjuvant Containing Two Delivery Systems with the Ability to Induce Mucosal Immunoglobulin A Following a Parenteral Immunization
}

Dennis Christensen, ${ }^{\dagger}$ Lasse Bøllehuus Hansen, ${ }^{\dagger}$ Romain Leboux, ${ }^{\dagger},{ }^{\S}$ Wim Jiskoot, ${ }^{\S}$ Jan Pravsgaard Christensen, "Peter Andersen, ${ }^{\dagger}$ and Jes Dietrich*, ${ }^{*} \odot$

${ }^{\dagger}$ Department for Infectious Disease Immunology, Statens Serum Institut, Artillerivej 5, DK-2300 Copenhagen, Denmark

${ }^{\ddagger}$ Department of Growth and Reproduction, Rigshospitalet, Juliane Maries Vej 6, DK-2100 Copenhagen, Denmark

${ }^{\S}$ Division of Bio-therapeutics, Leiden University, Einsteinweg 55, NL 2333 Leiden, Holland

"Department of Immunology and Microbiology, University of Copenhagen, Blegdamsvej 3C, DK-2200 Copenhagen, Denmark

Supporting Information

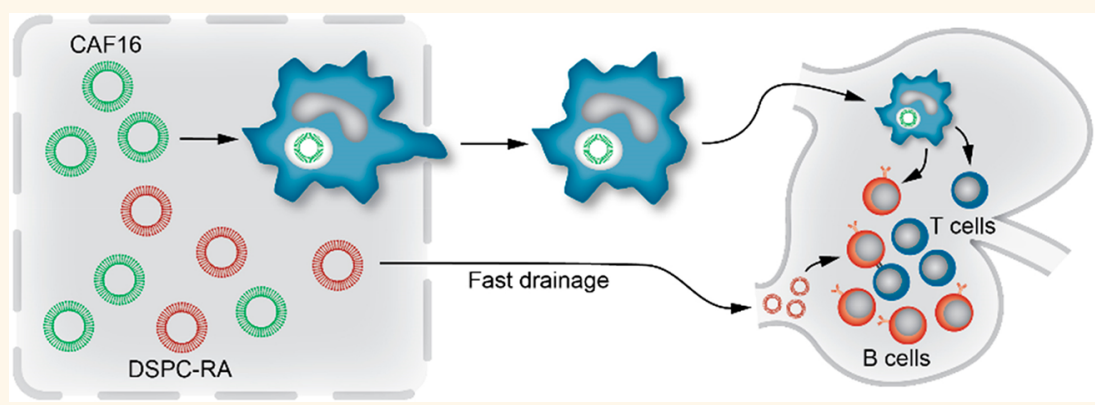

ABSTRACT: Worldwide, enteric infections rank third among all causes of disease burdens, and vaccines able to induce a strong and long-lasting intestinal immune responses are needed. Parenteral immunization generally do not generate intestinal IgA. Recently, however, injections of retinoic acid (RA) dissolved in oil, administered multiple times before vaccination to precondition the vaccine-draining lymph nodes, enabled a parenteral vaccine strategy to induce intestinal IgA. As multiple injections of RA before vaccination is not an attractive strategy for clinical practice, we aimed to develop a "one injection" vaccine formulation that upon parenteral administration induced intestinal IgA. Our vaccine formulation contained two liposomal delivery systems. One delivery system, based on 1,2-distearoyl-sn-glycero-3-phosphocholine stabilized with PEG, was designed to exhibit fast drainage of RA to local lymph nodes to precondition these for a mucosal immune response before being subjected to the vaccine antigen. The other delivery system, based on the cationic liposomal adjuvant CAF01 stabilized with cholesterol, was optimized for prolonged delivery of the antigen by migratory antigen-presenting cells to the preconditioned lymph node. Combined we call the adjuvant CAF23. We show that CAF23, administered by the subcutaneous route induces an antigen specific intestinal IgA response, making it a promising candidate adjuvant for vaccines against enteric diseases.

KEYWORDS: vaccine, mucosa, IgA, intestine, adjuvant

$\mathrm{M}$ ost pathogens as well as environmental allergens invade or enter the host through mucosal surfaces. Worldwide, enteric infections rank third among all causes of disease burden, and vaccines with the ability to induce a strong and long-lasting intestinal immune response are therefore needed. ${ }^{1}$

Due to the lack mucosal adjuvants, few mucosal vaccines are available for human use. Common for most enteric infections is that they require a robust mucosal immune response, composed of $\operatorname{IgA}$, to combat them. One way to induce intestinal immunity has been with vaccines delivered by the oral route. However, most antigens are not very immunogenic when administered via the oral route. ${ }^{2}$ Although safe, easy, and efficient, systemic immune responses induced by classical

Received: July 10, 2018

Accepted: January 4, 2019

Published: January 4, 2019 


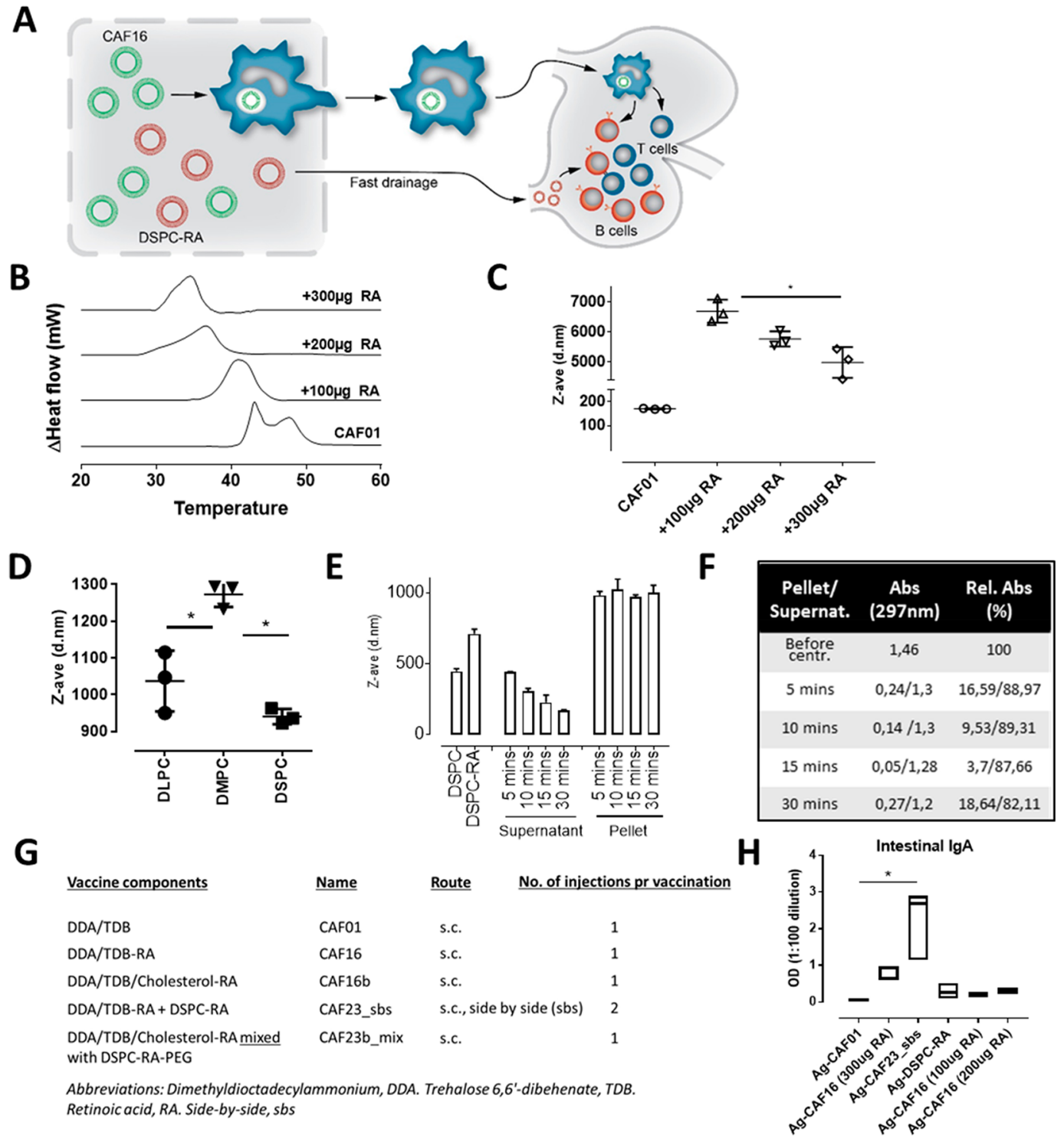

Figure 1. CAF23 adjuvant and its components. (A) Illustration showing the hypothesis behind the vaccine delivery strategy. CAF16, which contains RA (green), generates a depot at the site of injection targeting the migratory APCs, while simultaneous injection of the fast draining DSPC-RA formulation (red) conditions lymph node resident cells to facilitate a mucosal IgA response. (B) Differential scanning heat capacity curves obtained at a scan rate of $30^{\circ} \mathrm{C} / \mathrm{h}$ for freshly prepared CAF01 liposomes incorporating $0,100,200$, and 300 $\mu \mathrm{g}$ of RA. The second of three DSC heating scans is shown. (C) Average particle size of CAF01 liposomes containing 0, 100, 200, and 300 $\mu \mathrm{g}$ of RA. The liposomes were dispersed in $10 \mathrm{mM}$ Tris buffer adjusted to $\mathrm{pH}$ 7.4. (D) Average particle size of liposomes based on DLPC, DMPC, and DSPC containing $300 \mu \mathrm{g}$ of RA. The liposomes were dispersed in $10 \mathrm{mM}$ Tris buffer adjusted to $\mathrm{pH}$ 7.4. (E) Average particle size of pellets and supernatant of a DSPC-RA liposome dispersion after 0, 5, 10, 15, and $30 \mathrm{~min}$ of centrifugation at $10000 \mathrm{~g}$ in Eppendorph vials. (F) Percentage of total DSPC-RA in pellets/supernatant (right column) after centrifugation as described above. (G) Nomenclature for the adjuvants tested in ensuing studies. (H) IgA titer in intestines following three vaccinations with indicated adjuvants. $*$ denotes $p<0.05$, oneway ANOVA, Tukey's multiple comparisons test.

parenteral injection routes are not the optimal method to induce immunity against a pathogen colonizing the intestines. However, due to the general safety and efficacy profiles of these administration routes, we nevertheless aimed to develop a formulation that enabled the induction of both systemic and local intestinal immunity. To this end, we modified a cationic liposomal-based adjuvant, developed to induce systemic immunity, to also induce intestinal immunity, when given as a parenteral vaccine.

Vitamin A is an essential micronutrient with the ability to regulate immunity at mucosal surfaces. ${ }^{3,4}$ Most of the immunological functions of vitamin A depend on its metabolite retinoic acid (RA), principally all-trans-RA and 9cis-RA. ${ }^{4}$ Previous studies showed that RA can influence $\mathrm{CD} 4^{+}$ $\mathrm{T}$ cell immunity and differentiation toward Th1/Th17 polarization as well as the homeostasis of dendritic cells (DCs). ${ }^{4-6}$ Importantly for this study, RA also increases mucosal homing capacity of $\mathrm{T}$ and $\mathrm{B}$ cells and facilitates induction of IgA-producing plasma cells. ${ }^{3,7-10}$ This capability makes RA an interesting component for the development of vaccines against enteric diseases. Several studies in animal models have shown that treatment with RA before or during oral vaccination enhanced mucosal immunity (reviewed in ref 11). Furthermore, a recent study showed that oral pretreat- 
ment of RA significantly reduced outer membrane vesicle (OMV)-induced pro-inflammatory responses after oral vaccination and increased mucosal immunity, but at the cost of systemic immunity. ${ }^{12}$ In a human clinical study in Zambia, in which men received oral RA 1 hour before and for 7 days following an oral typhoid vaccine, it was shown that RA treatment can increase antigen-specific IgA responses to the vaccine in the gut. With the vast majority of vaccines being applied by a parenteral route, this route is also attractive for RA administration. However, the poor aqueous solubility of RA makes it difficult to be formulated into a parenteral formulation. In order to circumvent this, RA has been dispersed into solvents with surfactant properties, such as DMSO, ${ }^{13}$ polymers, ${ }^{10}$ and emulsions. ${ }^{14}$ Thus, Hammerschmidt et al. showed that s.c. vaccination in combination with s.c. injection of RA at day 0,1 , and 3 facilitated increased induction of gut-homing receptors on effector cells and led to potent intestinal immune responses, which protected the mice against an oral infection with salmonella. ${ }^{10}$

The conditioning of the immune system both before and after vaccination makes it a difficult vaccine strategy to apply in clinical practice. The purpose of the present study was to address this and to design a vaccine formulation that does not involve pre- and post-treatment of draining lymph nodes with $\mathrm{RA}$ in order to achieve intestinal IgA after immunization. We used the adjuvant CAF01 as backbone for the vaccine delivery system. ${ }^{15,16}$ CAF01 is composed of cationic liposomes based on the surfactant DDA (dimethyldioctadecylammonium bromide) stabilized with the synthetic immunostimulator TDB (trehalose 6,6'-dibehenate). ${ }^{17}$ CAF01 enhances both humoral and cell-mediated memory immune responses to several vaccine candidates ${ }^{18-20}$ and has been tested in phase I trials with excellent safety and immunogenicity profile. ${ }^{15,21-23}$ CAF01 forms a depot at the site of injection, thus targeting a narrow subset of migratory APCs. The concept was therefore to combine, in one single vaccine administration, the depotforming CAF01 delivery system with a fast draining delivery system (containing RA) that would condition the cells in the draining lymph node before being subjected to the antigen. We named the combined formulation CAF23, and we show here that CAF23 is able to induce antigen-specific intestinal IgA following parenteral administration.

\section{RESULTS AND DISCUSSION}

Proof of Concept for Supplementing CAF01 with Retinoic Acid. Previous studies showed that supplementing a parenteral vaccine with retinoic acid resulted in increased intestinal IgA. ${ }^{10,13}$ In these studies mice were pre- and/or posttreated with RA/oil (according to the schedule shown in Figure S1A) in order to condition the draining lymph node to generate lymphocytes with mucosal-homing potential. As an initial experiment we replicated these studies showing increased intestinal IgA by pre- and post-treating with 300 $\mu \mathrm{g}$ of $\mathrm{RA} /$ oil and immunizing with recombinant antigen formulated in the adjuvant CAF01 ${ }^{17,24}$ (Figure S1). However, considering the practical implications of this vaccine schedule, our idea was to generate two liposomal RA formulations. One formulation should be a fast draining formulation to condition the lymph node, and another formulation should be a depotforming formulation also containing the vaccine antigen. Importantly, the two formulations should be given simultaneously, as side-by-side immunization.
In the depot-forming formulation RA was incorporated into the cationic liposomal adjuvant CAF01 (named "CAF16"), whereas in the fast draining formulation, RA was incorporated into neutral liposomes. An illustration of the two formulations and their expected effect is shown in Figure 1A. All formulations used in this paper are shown in Figure 1G.

The incorporation of RA into the CAF01 formulation was performed by dissolving RA into the organic phase before solvent evaporation. Different concentrations of RA were incorporated into the lipid bilayers of the liposomes. Heat capacity curves in Figure 1B show that the differential scanning calorimetry (DSC) scan for CAF01 dispersed in $10 \mathrm{mM}$ Tris at $\mathrm{pH} 7.6$ is characterized by a broad gel-fluid phase transition with two main peaks at 43.2 and $47.8{ }^{\circ} \mathrm{C}$, respectively. This suggests that more than one cooperative heat transition occurs, due to changes in the local structure of the DDA bilayer, resulting in a lateral phase separation and formation of domains enriched in TDB. ${ }^{17}$ Introduction of increasing amounts of RA led to a gradual shift in $T_{\mathrm{m}}$ toward lower temperatures. This was also observed for DDA liposomes containing increasing amounts of TDB and other glycolipids. ${ }^{17,25}$ Overall this demonstrates that RA is incorporated into the hydrophobic core of the CAF01 bilayer, causing a change in the lipid chain packing, and that the thermotropic phase behavior of the CAF01 liposomes is RA concentration dependent.

Incorporation of RA into CAF01 increased the average particle size significantly to $>5 \mu \mathrm{m}$ irrespective of the RA dose (Figure 1C). This resulted in particle flocculation and eventually precipitation. The precipitate could however be redispersed after gentle shaking.

For particles to be self-draining to the lymph node, size plays an important role. The smaller the particles are, the easier the drainage, and the optimum size range for lymphatic drainage from the injection site has been reported to be 40-200 $n$. $^{26-28}$ Concerning the fast draining formulation, we therefore first tested different DxPCs with acyl backbones with varying length $(\mathrm{C} 12, \mathrm{C} 14$, and $\mathrm{C} 18)$. Figure $1 \mathrm{D}$ shows that 1,2-distearoyl-sn-glycero-3-phosphocholine (DSPC)-based particles were the most optimal with respect to particle size. However, the average particle size was still too high, being in the range of 700-1000 nm (Figure 1E). We therefore removed the biggest particles from the formulation by gentle centrifugation $(10000 g)$ in Eppendorf vials. As illustrated in Figure 1E, centrifugation for 15 and $30 \mathrm{~min}$ reduced the average particle size to 220 and $170 \mathrm{~nm}$, respectively. Relative absorbance at $297 \mathrm{~nm}$ was used to quantify RA after centrifugation showing that the residual content after centrifugation was $87 \%$ after $15 \mathrm{~min}$ and $82 \%$ after $30 \mathrm{~min}$ (Figure 1F). We decided to continue with DSPC-RA (with centrifugation for $15 \mathrm{~min}$ ) in the next studies.

As the objective was to generate an adjuvant with the ability to induce mucosal IgA, we initially compared the CAF01 adjuvant with the adjuvants CAF16 (CAF01-RA) and CAF23 (CAF16 + DSPC-RA) (see Figure 1G for the nomenclature of the adjuvant formulations). Mice were vaccinated subcutaneously with recombinant antigen (here we used the Chlamydia trachomatis MOMP antigen) formulated in CAF01, CAF16, or CAF16 administered side-by-side with (i.e., in close proximity to) DSPC-RA to ensure draining to the same lymph node (LN) (CAF23_sbs). Note that none of the vaccines used any pre- or post-treatment with RA, but that CAF23_sbs consisted of two formulations injected separately, 


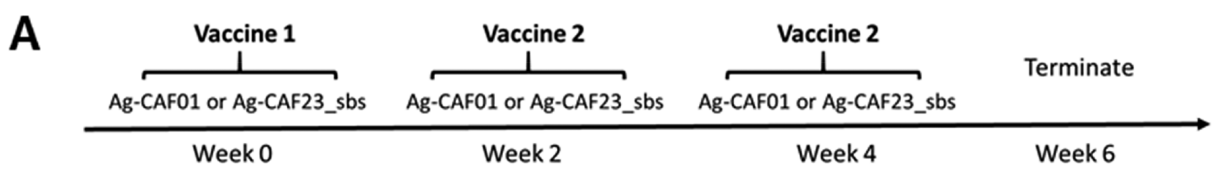

B
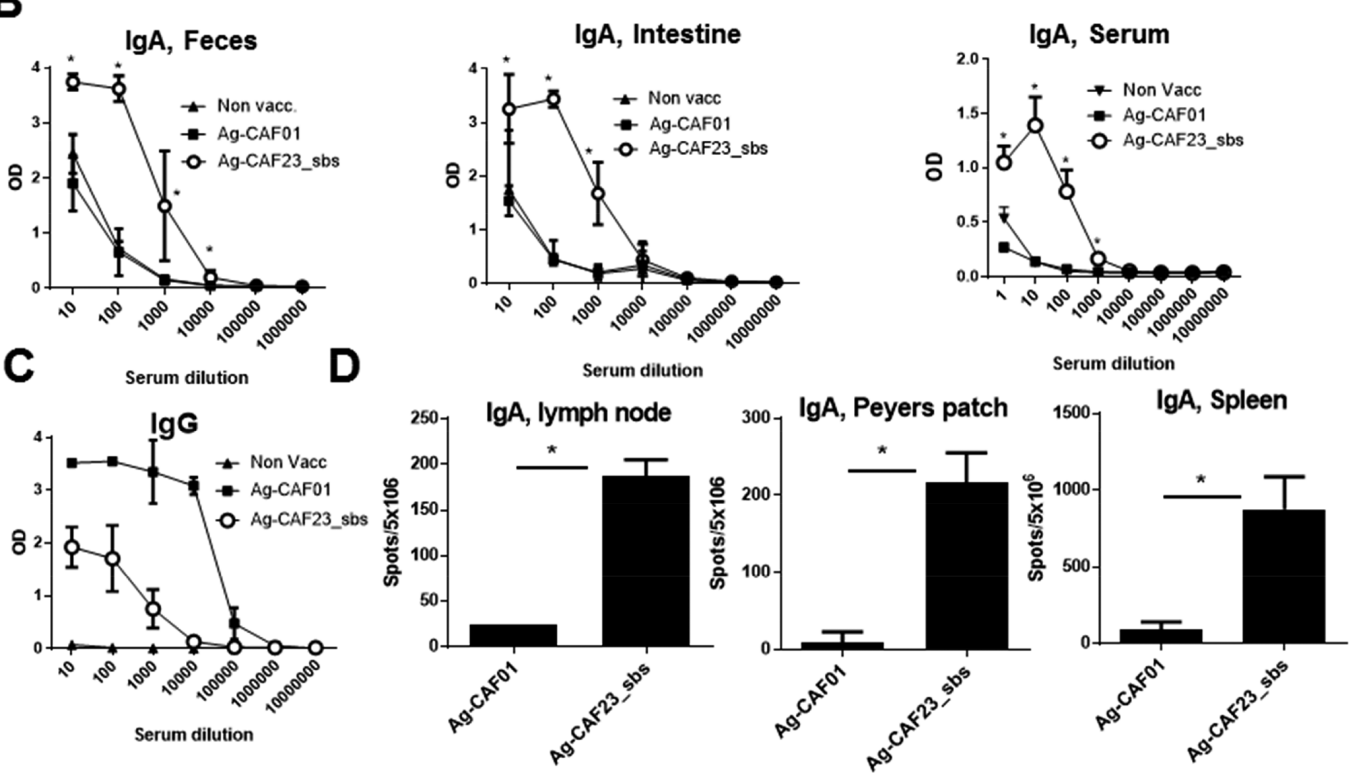

Figure 2. Humoral immune response following immunization with Ag-CAF23. (A) Overview of the immunization schedule. The antigen was MOMP. (B and C) Two weeks after the second vaccination, IgA was measured by indirect ELISA in intestines, feces, and blood (graph consists of data from two individual experiments, $n=16$ ). Graph shows mean \pm SD. (D) Cells from Peyer's patch, inguinal lymph node, and spleen were isolated, and the number of antigen-specific B cells were measured by ELIspot (duplicate samples from a pool of cells, $n=8$ ). Graph shows number of cells per $5 \times 10^{6}$ cells (mean \pm SD). In $B$, * denotes $p<0.05$, one-way ANOVA, Tukey's multiple comparisons test. In $\mathrm{D}, *$ denotes $p<0.05$, Student's $t$-test.

but simultaneously. Animals were vaccinated three times at two-week intervals, and IgA levels were determined in the intestines. The data showed that the highest IgA titers were observed in the group vaccinated with CAF23 sbs (Figure $1 \mathrm{H})$. CAF01 + DSPC-RA also induced IgA at slightly reduced levels (Figure S2), but we decided to continue with CAF23_sbs. Amounts of 100, 200, and $300 \mu \mathrm{g}$ of RA were tested in CAF01, but as 100 and $200 \mu \mathrm{g}$ (in contrast to 300 $\mu \mathrm{g}$ ) did not lead to elevated IgA titers in the intestines (Figure $1 \mathrm{H}$ ), we used $300 \mu \mathrm{g}$ of RA in the CAF23 sbs formulation and in the DSPC formulation. In agreement with this, in contrast to $300 \mu \mathrm{g}$ of RA, 100 and $200 \mu \mathrm{g}$ of RA administered in oil next to Ag-CAF01 also did not induce an increased IgA response compared to Ag-CAF01 (Figure S1).

Humoral Response Induced by CAF23. To test the immunological properties of CAF23_sbs, mice were vaccinated subcutaneously with Ag admixed with CAF01 or CAF23_sbs (vaccine schedule is shown in Figure 2A). We first examined the IgA response in the intestines and feces 2 weeks following the last vaccination. The fecal pellets were included, as blood contamination is expected to be less compared to intestinal samples. Fecal pellets were taken from the colon of sacrificed animals. The results showed increased IgA levels in both feces and intestinal samples from Ag+CAF23 sbs-vaccinated animals compared with Ag+CAF01-vaccinated animals (Figure 2B). In addition, we observed increased serum IgA levels in Ag + CAF23 sbs-vaccinated animals (Figure 2B).

Ag+CAF23_sbs-immunized mice showed a reduced IgG titer in serum as compared with $\mathrm{Ag}+\mathrm{CAF01-immunized} \mathrm{mice}$ (Figure 2C). This was in agreement with the results obtained after pre- and post-treating with RA in oil (Figure S1B-D). Analysis of the individual IgG subsets (in a subsequent experiment) revealed that $\operatorname{IgG} 2$ isotypes were reduced, but not IgG1. This pattern was observed in the serum, intestine, and feces (Figure S3). In addition to increased levels of IgA, Ag +CAF23_sbs-vaccinated animals also experienced increased numbers of Ag-specific B cells in Peyer's patches, draining inguinal lymph nodes and the spleen, compared with $\mathrm{Ag}$ +CAF01-vaccinated animals (Figure 2D).

In summary, we were able to incorporate RA into both CAF01 and DSPC to generate the CAF23 adjuvant. As a parenteral adjuvant, CAF23 sbs was able to generate antigenspecific mucosal IgA in the intestine and feces. This was accompanied by lower IgG2 levels.

Optimizing the Stability of the CAF01-RA and DSPCRA Formulations. As both formulations in CAF23 showed some precipitation at storage, we next investigated whether the two formulations could be stabilized without compromising their immunological properties.

Optimizing the CAF16 Formulation. The incorporation of cholesterol into liposomal membranes leads to improved lipid packing, reduced main phase transition temperature, and increased bilayer fluidity, resulting in improved stability. ${ }^{29-31}$ Moreover, a previous study showed that incorporation of cholesterol into phospholipid-based liposomes increased both the incorporation efficiency and the stability of retinol. ${ }^{32}$

To test if this effect could be achieved with CAF16, we incorporated increasing concentrations of cholesterol ranging from $10 \%$ to $50 \%$ (w/w DDA). Visual inspection clearly documented an effect on stability by incorporation of 


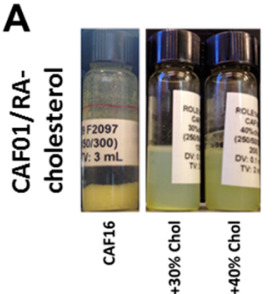

D

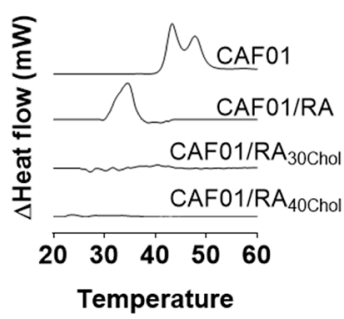

B

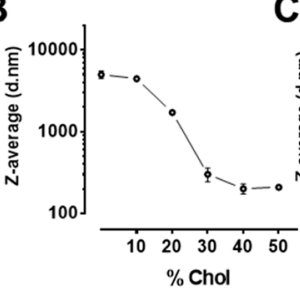

E
$\mathbf{F}$

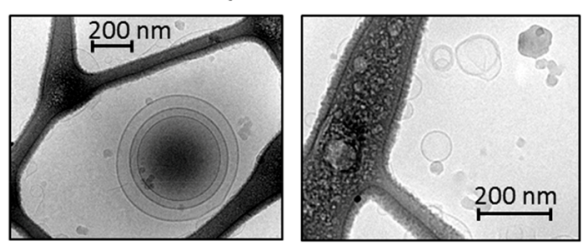

G

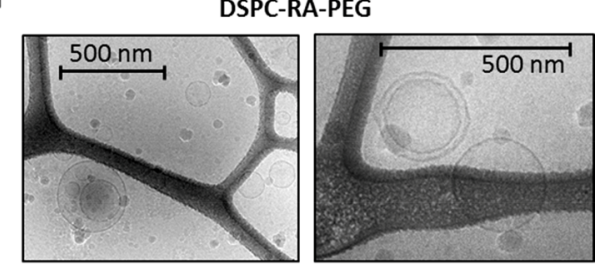

Figure 3. Optimizing the stability CAF16 and DSPC-RA. (A-C) Incorporation of cholesterol stabilized the CAF16 liposomes. (A) CAF16 without (left) and with $30 \%$ (middle) and $40 \%$ (right) cholesterol $24 \mathrm{~h}$ after formulation. (B) Average particle size of CAF16 liposomes containing $0,10,20,30,40$, and 50\% cholesterol. The liposomes were dispersed in $10 \mathrm{mM}$ Tris buffer adjusted to $\mathrm{pH}$ 7.4. Incorporation of increasing amounts of cholesterol results in a gradual reduction in CAF16 particle size. (C) Stability assessment over 8 weeks of CAF16 liposomes without $(\square)$ and incorporating $30 \%(\triangle)$ and $40 \%(\nabla)$ cholesterol as measured by average particle size. Particle size was benchmarked with CAF01 $(O)$. The liposomes were dispersed in $10 \mathrm{mM}$ Tris buffer adjusted to $\mathrm{pH}$ 7.4. (D) Differential scanning heat capacity curves obtained at a scan rate of $30{ }^{\circ} \mathrm{C} / \mathrm{h}$ for freshly prepared CAF16 liposomes with or without cholesterol. The second of three DSC heating scan is shown. (E) PEG coating stabilized the DSPC-RA liposomes. Stability assessment over 6 weeks (44 days) of DSPC-RA liposomes with or without incorporation of DSPE-PEG as measured by average particle size. The liposomes were dispersed in $10 \mathrm{mM}$ Tris buffer adjusted to $\mathrm{pH}$ 7.4. (F and G) Cryo-TEM pictures of CAF01/RA-cholesterol (CAF16b) (F) and DSPC-RA-PEG (G).
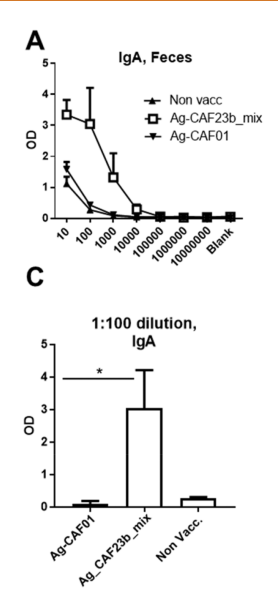
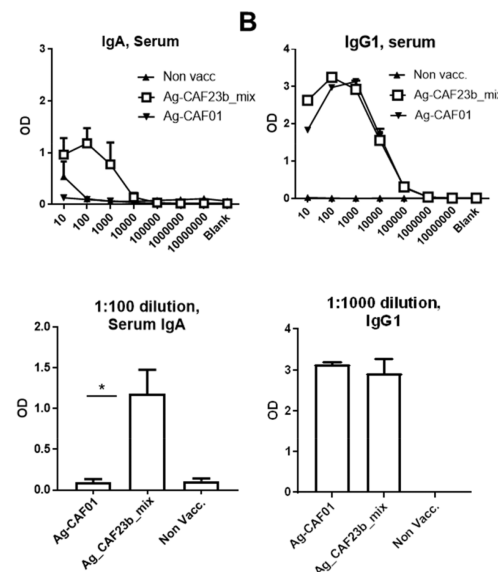
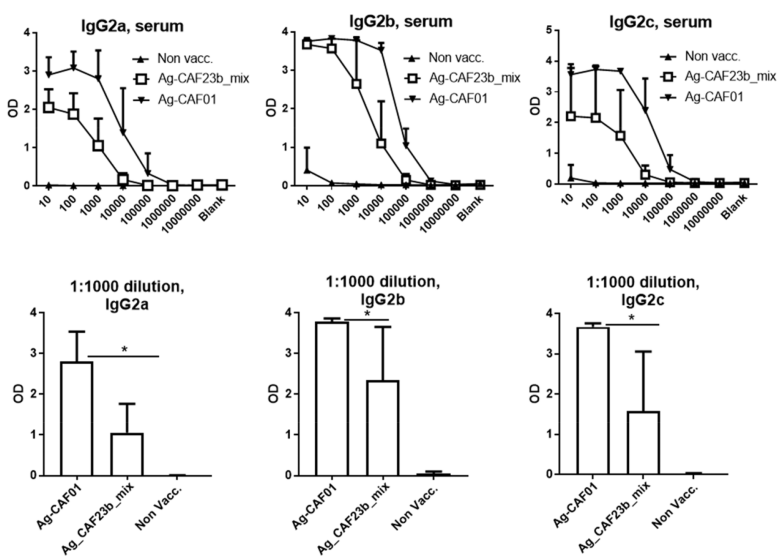

Figure 4. Immunological properties of CAF23b_mix. (A) Two weeks after the second vaccination IgA was measured by indirect ELISA in feces or serum $(n=4)$. (B) The serum IgG1, IgG $2 a$, IgG2b, and IgG2c against the vaccine antigen MOMP was measured by indirect ELISA $(n=8) 2$ weeks after the last vaccination. (C) OD values from 1:100 dilution (IgA) or 1:1000 dilution (IgG2a,b,c). * denotes $p<0.05$, oneway ANOVA, Tukey's multiple comparisons test.

cholesterol into CAF16, as no precipitation was observed after incorporation of $40 \%$ cholesterol (Figure 3A). Size and polydispersity were measured by dynamic light scattering. CAF16 particles were large with an average size above $4 \mu \mathrm{m}$ (Figure 1C). By addition of cholesterol, the average particle size gradually decreased, and incorporation of $40 \%$ cholesterol resulted in particles with an average particle size of $200 \mathrm{~nm}$, similar to that of CAF01. Incorporating more cholesterol did not decrease the size further (Figure $3 \mathrm{~B}$ ). CryoTEM pictures of CAF16 + 40\% cholesterol ("CAF16b") support these data and show that the CAF16b liposomes mainly consist of unilamellar and small onion-like multilamellar vesicles (Figure 3 F). Long-term stability studies showed that CAF16 with $40 \%$ cholesterol exhibited a stability comparable to CAF01 (Figure $3 \mathrm{C})$. As previously described for CAF01, ${ }^{31}$ the incorporation of cholesterol into CAF16 led to a gradual reduction in the main phase transition (Figure 3D), probably due to the formation of a liquid-ordered structure of lipid membrane below the phase transition of the DDA lipid bilayer, thereby eliminating the gel-liquid phase transition.

Optimizing the DSPC-RA Formulation. The main reason for optimizing the DSPC-RA formulation was to increase the long-term stability and to reduce the particle size. Poly(ethylene glycol) (PEG) is the most widely used hydrophilic polymer for the steric stabilization of liposome drug delivery systems. We chose to incorporate $15 \%$ (w/w) DSPEPEG2000. By this we obtained DSPC-RA liposomes with a long lasting stability, as illustrated in Figure 3E.

For the next experiment with CAF23, we selected CAF16b and the DSPC-RA formulation with 15\% DSPE-PEG2000. As 
A

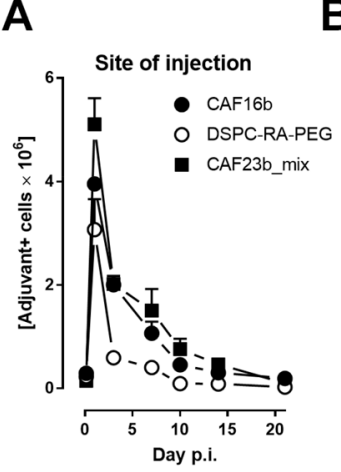

B

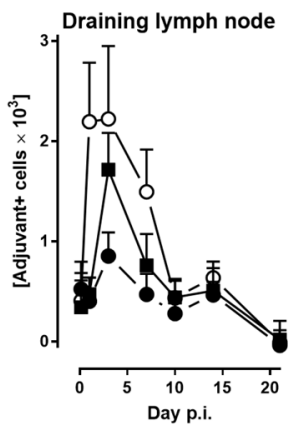

C
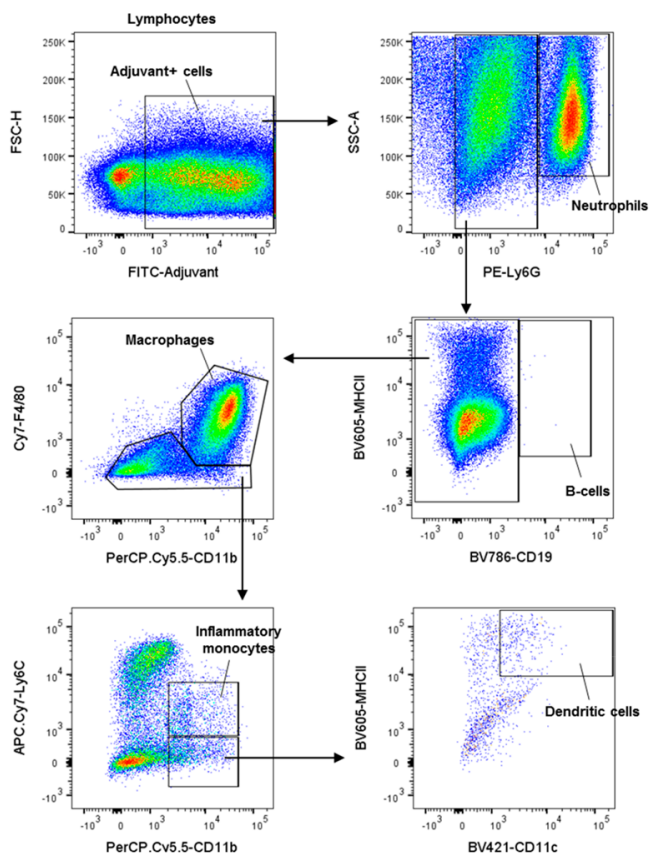

Site of injection

E
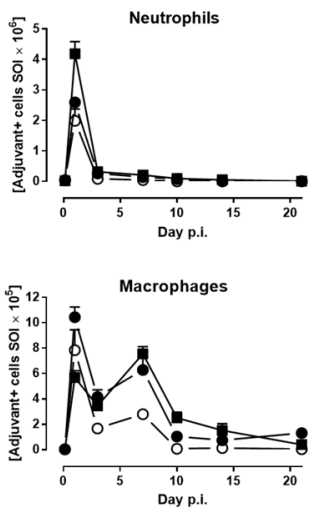

F

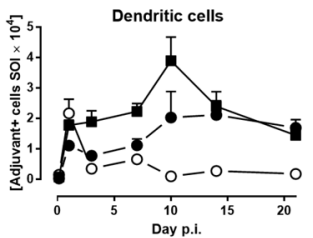

G

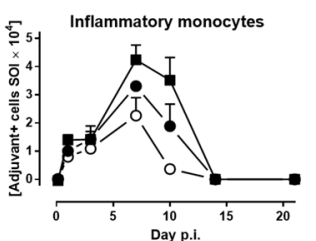

H

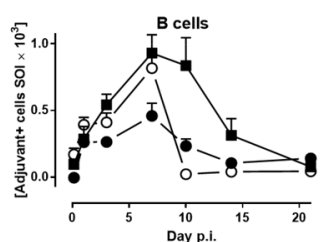

Draining LN
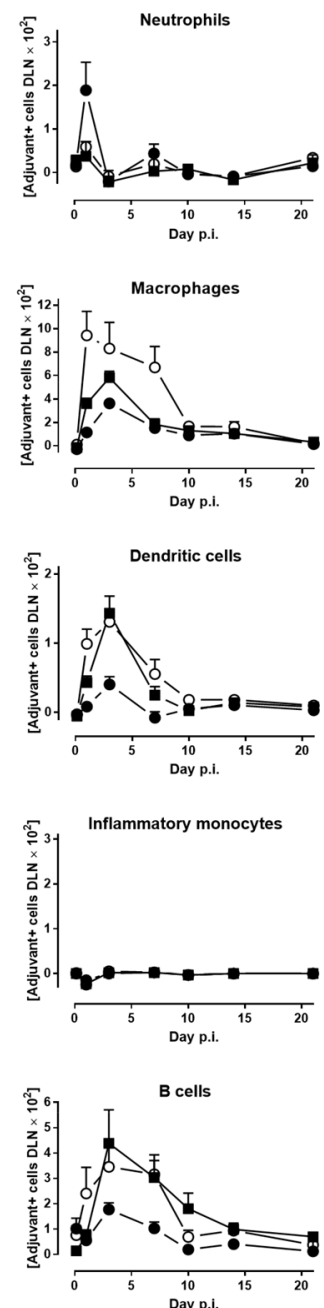

Figure 5. Biodistribution and cellular uptake of CAF23b adjuvant components. Qualitative association of fluorescently labeled vaccine components with lymphocytes was evaluated using flow cytometry. (A, B) Mice were immunized with vaccines containing fluorescently labeled CAF16b, DSPC-RA, or CAF23b_mix, and the percentage of adjuvant+ lymphocytes at the site of injection (A) and in the draining LN (B) was evaluated. The antigen in CAF16b and CAF23b_mix formulations was OVA, $5 \mu \mathrm{g}$ per dose. Data points represent mean \pm SEM $(n=5)$. (C) The adjuvant + lymphocytes were divided into neutrophils $($ Ly6G +$)$, B cells (Ly6G ${ }^{-}$CD 19 $\left.^{+}\right)$, macrophages

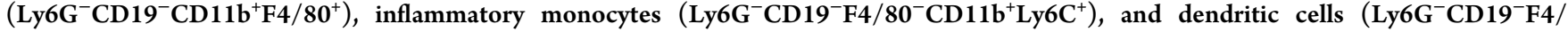

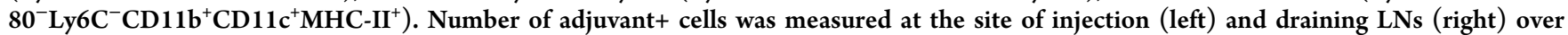
time for (D) neutrophils, (E) macrophages, (F) dendritic cells, (G) inflammatory monocytes, and (H) B cells. (D-H) Data points represent $n=5$ and shows mean \pm SD.

described for DSPC-RA, larger particles were removed from the formulation by centrifugation for $15 \mathrm{~min}$. Cryo-TEM pictures of DSPC-RA-PEG showed that the liposomes were very similar to CAF16b (Figure $3 \mathrm{~F}$ and $\mathrm{G}$ ). They primarily contained unilamellar and small multivesicular vesicles (Figure $3 \mathrm{G})$. The multivesicular vesicles did not always take an onionlike shape, but instead other multivesicular forms, as illustrated in the left picture in Figure 3G.

Immunological Properties of CAF23b_mix. In clinical practice, one single formulation is preferred over two formulations, even though the two formulations can be administered simultaneously. We therefore tested a "combined formulation" strategy where the CAF16b and the DSPC-RAPEG components were mixed ("CAF23b_mix") into the same vial prior to vaccination. CAF23b_mix showed the same immunological properties as CAF23 in terms of an increased mucosal IgA response, an unaltered IgG1 response, and a reduced IgG2a/IgG2b/IgG2c response (Figure 4A-C).

In summary, without compromising the immunological properties, we succeeded in optimizing the stability of the two vaccine components and showed that they can be administered as one combined vaccine formulation.

Biodistribution and Cellular Uptake of the Vaccine Components. The idea behind CAF23 was to design an adjuvant with a fast and slow draining component. In order to validate the hypothesis, we investigated the distribution and cellular association at the site of injection (the muscle) and in the draining inguinal lymph node (dLNs) of 3,3'-dioctadecyloxacarbocyanine (DiO)-labeled DSPC-RA-PEG and CAF16b for 21 days following an i.m. administration. These analyses were performed in animals immunized with the individual components or with CAF23b_mix. 
Immunization with CAF16b established a depot at the site of injection with significant levels of adjuvant positive (adjuvant + ) cells detectable from day 1 postimmunization to as long as 21 days postimmunization (Figure 5A). This was not observed with DSPC-RA-PEG. Thus, already after 3 days DSPC-RA-PEG-immunized mice showed significantly lower numbers of adjuvant+ cells at the site of injection compared to CAF16b-immunized mice. In the LN the pattern was reversed: DSPC-RA-PEG immunization resulted in a rapid cellular localization of the vaccine component, whereas in CAF16b mice adjuvant+ cells were not detectable in lymphocytes in the dLN until day 3 and still in much lower numbers (Figure 5B).

Interestingly both a depot effect and a fast draining to the LNs were observed with CAF23b_mix, indicating that mixing the two components into one vaccine formulation does not compromise the properties of the components.

We also investigated the phenotype of the vaccine positive (adjuvant+) cells in the two locations (see gating strategy in Figure $5 \mathrm{C}$ ). At the site of injection there was a rapid influx of neutrophils $\left(\mathrm{Ly} 6 \mathrm{G}^{+}\right)$, which however disappeared after only 3 days (Figure 5D, right side). These neutrophils did not reach the dLNs (Figure 5D left). Adjuvant+ macrophages were detected from day 1 after injection and throughout the rest of the experiment in mice receiving the depot-forming adjuvants CAF16b and CAF23b_mix, whereas detectable adjuvant+ macrophages were not detectable after 10 days in DSPC-RAPEG mice (Figure 5E left). In contrast, in the draining LNs, DSPC-RA-PEG mice (and to lesser extent CAF23b mix mice) showed elevated levels of adjuvant+ macrophages, compared to CAF16b (Figure 5E, right side).

The rapid localization of adjuvant+ cells in DSPC-RA-PEG and CAF23b_mix mice in the dLNs also resulted in a simultaneous rapid association of the vaccine with the DCs $\left(\mathrm{CD} 11 \mathrm{~b}^{+} \mathrm{CD} 11 \mathrm{c}^{+} \mathrm{MHCII}^{+}\right.$, Figure 5F, right), whereas it was primarily the depot-forming CAF16b and CAF23b_mix that led to adjuvant+ DCs at the site of injection (Figure 5F, left). Finally, at the site of injection, the association with inflammatory monocytes ( $\left.\mathrm{CD} 11 \mathrm{~b}^{+} \mathrm{Ly} 6 \mathrm{C}^{\text {int }}\right)$ correlated with the ability to form a depot, whereas no inflammatory monocytes were observed in the dLNs (Figure 5G). B cell association was also observed, in particular with DSPC-RAPEG and CAF23 mix (Figure 5H).

In summary, the depot-forming formulation CAF $16 \mathrm{~b}$ showed less adjuvant+ cells in the dLNs, and at the site of injection the vaccine was associated with neutrophils (only initially), macrophages, dendritic cells, and inflammatory monocytes. In contrast, the fast draining formulations (DSPC-RA-PEG and CAF23b_mix) showed increased adjuvant+ cells in the dLNs, which was characterized as macrophages, dendritic cells, and B cells.

Although oral vaccines have been shown to induce intestinal immunity, studies in humans have indicated that oral vaccines show varying efficacy. ${ }^{33,34}$ A parenteral vaccine in general induces systemic immunity, but only to a minor degree mucosal immunity. One example is parenteral vaccines based on the adjuvant CAF01. ${ }^{17,24,35}$ Several studies have however shown that all-trans-retinoic acid (ATRA) dissolved in oil given before and during priming of an immune response with a parenteral vaccine can induce homing of lymphocytes to the intestines and subsequent increased intestinal $\operatorname{IgA}{ }^{10,13,36}$ These studies utilized several injections of RA, and in most cases RA was dissolved in oil (e.g., soybean oil or olive oil). In the present study we initially used RA dissolved in olive oil to treat the lymph nodes before administering antigen formulated in CAF01. By this we were able to replicate previous results (now with CAF01) and show increased IgA levels in the intestines due to the addition of RA (Figure S1). This prompted us to develop an RA-containing adjuvant system, which is more clinically relevant than having to pretreat with RA dissolved in oil.

Liposomes can serve as carriers for the poorly soluble retinoic acid, and the biological activity of RA is not compromised by being incorporated into the liposomes. ${ }^{37,38}$ The idea was therefore to incorporate RA into the liposomal adjuvant CAF01 and combine this controlled delivery of antigen with the fast draining DSPC-RA formulation.

Our data showed that RA could be incorporated into the hydrophobic core of the CAF01 bilayer. However, some precipitation was observed with this formulation. The incorporation of cholesterol into liposomal membranes has been shown to lead to improved stability ${ }^{29-31}$ and to increase the incorporation of retinol. ${ }^{32}$ In agreement with these studies, we showed that addition of $40 \%$ cholesterol resulted in particles with an average particle size of $200 \mathrm{~nm}$ and significantly improved stability of the formulation (Figure 3). Cholesterol also led to a reduction in the main phase transition, similar to what has previously been described for CAF01. ${ }^{31}$ Taken together, incorporation of RA and cholesterol into CAF01 liposomes generated an adjuvant with a physical stability appropriate for a vaccine.

The other component of CAF23, DSPC-RA, was optimized with PEG, which is known to induce steric stabilization of liposomal drug delivery systems and used in a number of marketed products including Doxil/Caelyx (a PEGylated liposomal delivery system for doxorubicin). It has also been shown that there is a direct correlation between the PEG grafting density and the degree of lymphatic drainage after grafting liposomes with 5-25\% DSPE-PEG2000. ${ }^{39}$ We therefore tested PEG and found that by incorporating $15 \%$ (w/w) DSPE-PEG2000 we obtained DSPC-RA liposomes with a long-lasting stability (Figure 3E).

Regarding the humoral immune response, the effect of adding RA was an increase in the IgA titer in intestine, feces, and blood and an increase in the IgG1/IgG2 ratio (due to a reduction in the IgG2 titers), as exemplified by CAF16 (Figure 1), CAF23 (Figure 1-3), or CAF23b_sbs/mix (Figure 5). This is in agreement with previous studies where ATRA supplementation promoted a Th2 phenotype. ${ }^{40-42}$ Whether this change in IgG1/IgG2 ratio is important for promoting an IgA response is not known.

We also observed a reduction in the total IgG response against the vaccine antigen. In contrast, other previous studies showed an RA-induced increase in the IgG titer. In several of these studies RA acted in synergy with TLR receptors, e.g.,

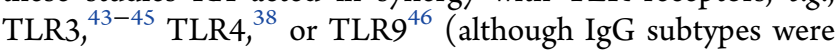
not measured in these studies). In one study RA was also used together with polyriboinosinic:polyribocytidylic acid and was able to increase all IgG subtypes (IgG1, IgG2a, and IgG2b), ${ }^{47}$ in contrast to our observations with CAF01 and RA. As CAF01 signals through the Mincle receptor, ${ }^{48}$ the effect of RA may be dependent on a specific crosstalk between the receptor for RA and signaling through other receptors on the APC. Indeed, it has been suggested that a crosstalk exists between RA and MyD88-dependent pathways. ${ }^{49}$ Whether a specific crosstalk exists between RA- and the Mincle-dependent pathway, known to use Syk-CARD9-dependent signaling, is not known. 
In agreement with previous studies ${ }^{10} \mathrm{RA}$ induced antigenspecific IgA $\mathrm{A}^{+} \mathrm{B}$ cells in inguinal LNs, spleen, and Peyer's patch. This correlated with increased IgA levels in the intestines/feces and blood. Other studies showed that RA upregulated the $\mathrm{RADLH}$ enzyme in DCs, causing them to induce gut-homing potential (via the CCR9/ $\alpha 4 \beta 7$ receptors) of local lymphocytes. $^{7,9,50}$ We did not examine this in vivo, but did find that DSPC-RA-PEG upregulated the $\alpha 4 \beta 7$ receptor on $\mathrm{CD} 3 / \mathrm{CD} 28$ (or PMA/ionomycin)-stimulated CD4 or CD8 splenocytes in vitro and on $\mathrm{B}$ cells (data not shown).

The increased B cell response observed in the draining LNs, spleen, and the Peyer's patches indicated that RA was able to accelerate B lineage lymphoid differentiation and cause an IgA isotype shift. Such an acceleration of B cell differentiation has also been observed in other studies. ${ }^{40,51,52}$ It could be speculated that the effect of RA on B cell differentiation occurs through an enhanced germinal center formation, as previously suggested with the RA-induced increase in a tetanus toxoid vaccine response. ${ }^{53} \mathrm{We}$ are presently investigating this possibility.

Supplementing CAF16b with a fast draining delivery system (DSPC-RA-PEG) showed increased adjuvant+ cells in the dLNs. These were identified as macrophages, dendritic cells, and B cells. The direct interaction of RA on DCs has been reported to induce DCs with the ability to generate lymphocytes with gut-homing potential. ${ }^{10,36}$ This might explain the observed IgA levels in the intestines. RA has also been reported to directly induce intestinal-homing markers on $\mathrm{B}$ cells through their expressed RA receptor $\alpha$, and this may also be important in generating an effective gut humoral IgA response. ${ }^{54}$ In addition CAF23 may also induce IgA in other mucosal sites. Indeed, CAF23 also induced increased IgA levels in the vagina.

\section{CONCLUSIONS}

In conclusion, we have generated a stable adjuvant formulation with the ability to generate mucosal IgA when administered as a parenteral vaccine. The CAF23b formulation was composed of two delivery systems with different drainage/delivery kinetics. The two delivery systems could be mixed prior to administration into one combined adjuvant formulation.

\section{METHODS/EXPERIMENTAL}

Ethics Statement. Experiments were conducted in accordance with the regulations set forward by the Danish Ministry of Justice and animal protection committees by Danish Animal Experiments Inspectorate Permit 2004-561-868 (of January 7, 2004) and in compliance with European Community Directive 86/609 and the U.S. Association for Laboratory Animal Care recommendations for the care and use of laboratory animals. The experiments were approved by the Statens Serum Institut IACUC. The method of sacrifice was cervical dislocation.

Animal Handling. Studies were performed with 6- to 8-week-old female CB6F1 (C57BL/6xBALB/c) mice from Envigo, Scandinavia. Animals were housed in appropriate animal facilities at Statens Serum Institut.

Vaccine Formulation. The CAF01, CAF01-RA, and CAF01-RAcholesterol adjuvant formulations were manufactured with fixed DDA and TDB concentrations of 2500 and $500 \mu \mathrm{g} / \mathrm{mL}$, respectively. RA was added in concentrations of $0,1000,2000$, and $3000 \mu \mathrm{g} / \mathrm{mL}$, and cholesterol in concentrations of 1280 (30\%) and $2000 \mu \mathrm{g} / \mathrm{mL}(40 \%)$. The DSPC, DLPC-RA, DMPC-RA, DSPC-RA, and DSPC-RA-PEG adjuvant formulations were manufactured with $3000 \mu \mathrm{g} / \mathrm{mL} \mathrm{RA}$ and $1000 \mu \mathrm{g} / \mathrm{mL}$ DSPE-PEG2000 incorporated into liposomes based on a fixed molar dose of $3.2 \mu \mathrm{mol} / \mathrm{mL}$ DxPC (resembling $2500 \mu \mathrm{g} / \mathrm{mL}$
DSPC). In brief, a lipid film for $8 \mathrm{~mL}$ of adjuvant was formed by dissolving the lipid components in chloroform/methanol (9:1, v/v) and mixing in the right proportions. The organic solvent was removed using a gentle stream of $\mathrm{N}_{2}$, forming a thin lipid film at the bottom of the vial. The lipid film was hydrated to a total volume of $8 \mathrm{~mL}$ with 10 $\mathrm{mM}$ Tris-buffer, $\mathrm{pH}$ 7.6, by high-shear mixing (Heidolph Silent Crusher M) at $60-65{ }^{\circ} \mathrm{C}$ for $15 \mathrm{~min}$. Adjuvants were stored at $2-8$ ${ }^{\circ} \mathrm{C}$ until use. All vaccine formulations were prepared in LAF units. When RA was dissolved in oil, sterile filtered olive oil was used.

Fluorescently labeled liposomes were prepared as described above with the fluorescent label $\mathrm{DiO}$ (ThermoScientific, Waltham, MA, USA). The final dose was $250 / 50 / 300 / 200 / 0.002 \mu \mathrm{g}$ of DDA/TDB/ $\mathrm{RA} /$ cholesterol/DiO (CAF16b) or 250/100/300/0.002 $\mu \mathrm{g}$ of DSPC/ DSPE-PEG2000/DiO (DSPC-RA-PEG) in $100 \mu \mathrm{L}$ of isotonic, 9\% $(\mathrm{w} / \mathrm{v})$ trehalose $10 \mathrm{mM}$ Tris buffer.

Zeta Potential and Particle Size Distribution by DLS. The Zaverage diameter of the liposomes was determined by dynamic light scattering. The measurements were performed at $25{ }^{\circ} \mathrm{C}$ using a Malvern nanoZS (Malvern Instruments, Worcestershire, UK) equipped with a $633 \mathrm{~nm}$ laser and $173^{\circ}$ detection optics. Malvern DTS v. 5.10 software was used for data acquisition and analysis. Nanosphere size standards $60 \mathrm{~nm}$ (Duke Scientific Corp., Duke, NC, USA) were used to verify the performance of the instrument. The samples were diluted with $10 \mathrm{mM}$ Tris-buffer at $\mathrm{pH} 7.4$ to achieve the optimal vesicle concentration. The zeta-potential of the vesicles was measured at $25{ }^{\circ} \mathrm{C}$ using a Malvern nanoZS (using a monomodal analysis model) (Malvern Instruments) of a 1/10-1/400 dilution in milli-Q water.

Differential Scanning Calorimetry Analysis. The heat capacity of the liposomes was determined using a Micro DSCIII CSEvol (Setaram). An $800 \mu \mathrm{L}$ amount of undiluted sample was loaded in a standard Hilleroy metal cell. The control cell was filled with the same weight of $10 \mathrm{mM}$ Tris buffer ( $\mathrm{pH} 7.4$ ). Samples were heated from 20 to $80{ }^{\circ} \mathrm{C}$ at a scanning rate of $0.5{ }^{\circ} \mathrm{C} / \mathrm{min}$, and thermograms were obtained and analyzed using supplied software. The first of two scans of each sample was used for data analysis.

CryoTEM Pictures. The morphology of the liposomes was investigated by cryo-transmission electron microscopy (cryo-TEM) using a Tecnai G2 T20 TWIN transmission electron microscope (Thermo Fischer, USA) mounted with a $4 \times 4 \mathrm{~K}$ charged-coupled device Eagle camera from Thermo Fischer. CAF16b and DSPC-RAPEG samples for cryo-TEM were prepared under controlled temperature $\left(4{ }^{\circ} \mathrm{C}\right)$ and humidity conditions $(100 \%)$ within an environmental vitrification system using a Thermo Fischer Vitrobot Mark IV. A small sample droplet $(3 \mu \mathrm{L})$ was deposited onto a glowdischarged 300 mesh holey carbon grid. Excess liquid was removed by plotting and immediately plunged into liquid ethane, resulting in the formation of a thin $(10-500 \mathrm{~nm})$ vitrified film, which extended in the holes of the carbon film. The samples were kept at a liquid nitrogen temperature of $-174{ }^{\circ} \mathrm{C}$ and subsequently transferred to a Gatan 626 cryo-holder for imaging in the electron microscope. The sample temperature was continuously kept below $-180{ }^{\circ} \mathrm{C}$. All observations were made in the bright field mode at an acceleration voltage of 200 $\mathrm{kV}$. Digital images were recorded with a $4 \times 4$ CCD Eagle camera (Thermo Fischer).

Immunization and Antigen. Mice were immunized two or three times at two-week intervals s.c. with the indicated vaccine. The s.c. injections were given at the base of the tail. The doses used in the experiments are indicated in the figures. Two weeks after the last vaccination the immune response was analyzed.

The antigens used in the study were a recombinant chlamydia antigen, MOMP (major outer membrane protein), a vaccine candidate based on the MOMP protein called CTH522, or a recombinant tuberculosis fusion protein, Hybrid $1 .^{55,56}$

Measurements of Antibody Titers. Mice were bled for the collection of serum following vaccination. Maxisorp micro titer plates (Nunc, Maxisorp, Roskilde, Denmark) were coated with antigen (1 $\mu \mathrm{g} / \mathrm{mL}$ ) in phosphate-buffered saline (PBS) overnight at $4{ }^{\circ} \mathrm{C}$. Free binding sites were blocked with $2 \%$ skimmed milk in PBS. Individual mouse sera were analyzed in duplicate in 5-fold dilutions in PBS 
containing bovine serum albumin starting with a 100 -fold dilution. Horseradish peroxidase (HRP)-conjugated secondary antibodies (rabbit anti-mouse IgG, IgG1, IgG2a, IgG2b, IgG2c, and IgA; Zymed) diluted $1 / 2000$ in PBS with $1 \%$ bovine serum albumin. After $1 \mathrm{~h}$ of incubation, antigen-specific antibodies were detected by TMB substrate as described by the manufacturer (Kem-En-Tec, Copenhagen, Denmark). To stop the reaction, $100 \mu \mathrm{L}$ of $4 \mathrm{~N}$ sulfuric acid was added, and the optical density (OD) was measured at $450 \mathrm{~nm}$. The absorbance values were plotted as a function of the reciprocal dilution of serum samples.

Preparation of Fecal Pellets for Antibody Analysis. Fecal pellets were collected from mice 2 weeks following each immunization. The mice were placed in individual cages, and fresh fecal pellets ( 5 or 6 pellets per mouse) were collected into microfuge tubes containing $600 \mu \mathrm{L}$ of ice-cold buffer: PBS with soybean trypsin inhibitor (Sigma; $0.1 \mathrm{mg} / \mathrm{mL}$ ), bovine serum albumin (BSA; 1\% w/ v), ethylenediaminetetraacetic acid (EDTA; $25 \mathrm{mM})$, glycerol $(50 \%$ $\mathrm{v} / \mathrm{v}$ ), and phenylmethylsulfonylfluoride (PMSF; $1 \mathrm{mM}$ ) were used. The fecal pellets were broken up to form a suspension and then incubated on ice for $4 \mathrm{~h}$. After incubation, fecal pellet suspensions were clarified by centrifugation at $15500 \mathrm{~g}$ for $10 \mathrm{~min}$ at $4{ }^{\circ} \mathrm{C}$, and the supernatants transferred to microfuge tubes that had been blocked overnight with PBS containing $1 \%(\mathrm{w} / \mathrm{v})$ BSA. Supernatants were then frozen and analyzed at a later date by ELISA.

Preparation of Intestines for Antibody Analysis. Intestines were cut into $5-10 \mathrm{~mm}$ pieces and put into a buffer containing trypsin inhibitor $(0.1 \mathrm{mg} / \mathrm{mL}) / 50 \mathrm{mM}$ EDTA $/ 0.35 \mathrm{mg} / \mathrm{mL}$ Pefablock $/ 0.1 \mathrm{mg} / \mathrm{mL}$ BSA in PBS-Tween, $0.05 \% \mathrm{v} / \mathrm{v}$ ). Saponin/PBS was added to a final concentration of $2 \%$ and incubated at $4{ }^{\circ} \mathrm{C}$ overnight. Supernatants were collected after centrifugation for subsequent analysis of antibodies by ELISA.

Biodistribution Assessed by Fluorescent Labeling of the Vaccine Components. Mice were immunized with either CAF16b$\mathrm{DiO}$, DSPC-RA-PEG-DiO, or CAF23b-DiO. A naive group was also included as a negative control. The studies were repeated in three experiments. Mice were euthanized before (time point 0 ) or on day 1 , $3,7,10,14$, or 21 after the immunizations, and the inguinal lymph node (ILN) and injection side quadriceps muscles were removed. The LNs were treated with Liberase TL (Roche, Hvidovre, Denmark) to liberate the APCs from the LN collagen structure. Each LN was treated with $1.5 \mathrm{~mL}$ of RPMI 1640 supplemented with $10 \%(\mathrm{v} / \mathrm{v})$ heat-inactivated fetal calf serum, $5 \times 10-6 \mathrm{M} \beta$-mercaptoethanol, $1 \%$ (v/v) penicillin-streptomycin, $1 \%(\mathrm{v} / \mathrm{v})$ sodium pyruvate, $1 \mathrm{mM} \mathrm{L}$ glutamine, and $10 \mathrm{mM}$ HEPES (sRPMI) containing $3 \mu \mathrm{g}$ of DNase I and $30 \mu \mathrm{g}$ of Liberase. After $15 \mathrm{~min}$ of incubation at $37^{\circ} \mathrm{C}$ the LNs were passed through a nylon-mesh cell strainer, treated with $150 \mu \mathrm{L}$ of $100 \mathrm{mM}$ EDTA for $3 \mathrm{~min}$, and washed in ice-cold PBS. The muscles were treated with enzymes A, D, and P of the Skeletal Muscle Dissociation Kit (Miltenyi Biotec GmbH, Bergisch Gladbach, DE) according to the manufacturer's instructions. Cells from organs were resuspended in sRPMI, and for each $\mathrm{LN} /$ muscle $1 \times 10^{6}$ cells, or everything if the sample contained fewer cells, were transferred to a 96-well, V-bottomed plate and treated with Fc-block followed by fluorescent staining.

Flow Cytometry and Biodistribution Analysis. Cells were stained with combinations of the following antibodies: $\alpha$-Ly6C-APCCy7, $\alpha$-CD11b- PerCP-Cy5.5, $\alpha$-Ly6G-PE, $\alpha$-CD11c-BV421, $\alpha$ CD19-BV786, $\alpha$-I-A/I-E-BV605, $\alpha$-F4/80-PE-Cy7, $\alpha$-CD11c-BV421 (all from BD Bioscience). The stained cells were analyzed using a flow cytometer (BD LSRFortessa, BD Bioscience) and BD DIVA software (BD Bioscience), and the percentage of adjuvant+ lymphocytes at the site of injection and in the draining LN was evaluated. The adjuvant+ lymphocytes were divided into neutrophils (Ly6G+), B cells $\left(\mathrm{Ly}_{6 \mathrm{G}} \mathrm{CD}^{-} 9^{+}\right)$, macrophages $\left(\mathrm{Ly} 6 \mathrm{G}^{-} \mathrm{CD} 19^{-} \mathrm{CD} 11 \mathrm{~b}^{+} \mathrm{F} 4 / 80^{+}\right)$, in-

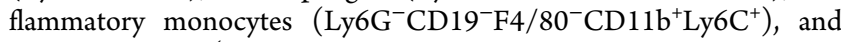
dendritic cells $\left(\mathrm{Ly}_{6 \mathrm{G}^{-}} \mathrm{CD} 19^{-} \mathrm{F} 4 / 80^{-} \mathrm{Ly} 6 \mathrm{C}^{-} \mathrm{CD} 11 \mathrm{~b}^{+} \mathrm{CD} 11 \mathrm{c}^{+} \mathrm{MHC}-\right.$ $\left.\mathrm{II}^{+}\right)$.

Statistical Methods. A difference of $p<0.05$ was considered significant using a one-way ANOVA and Tukey's multiple comparison test for multiple comparisons or Students $t$-test, as indicated in the figure text. Prism version 7 software (GraphPad) was used for analysis.

\section{ASSOCIATED CONTENT}

\section{S Supporting Information}

The Supporting Information is available free of charge on the ACS Publications website at DOI: 10.1021/acsnano.8b05209.

Immune responses when supplementing the CAF01 adjuvant with retinoic acid in an oil solution; IgA in feces, intestines, and serum after immunizing with CAF16 and CAF23; IgG1, IgG2a, IgG2b, and IgG2c titers after immunizing with CAF01 and CAF23 (PDF)

\section{AUTHOR INFORMATION}

\section{Corresponding Author}

*Fax: +45 326830 35. E-mail: jdi@ssi.dk (J. Dietrich). ORCID

Jes Dietrich: 0000-0001-8536-0141

\section{Author Contributions}

J.D. and D.C. designed the study. L.B. and R.L. performed most of the laboratory work. J.D., D.C., L.B., R.L., and P.A. analyzed the data. J.D., D.C., L.B., R.L., P.A., W.J., and J.C. interpreted the data. J.D. and D.C. drafted the manuscript.

Notes

The authors declare no competing financial interest.

\section{ACKNOWLEDGMENTS}

The excellent technical assistance provided by Lene Rasmussen and Janne Rabech and the animal technicians at the Statens Serum Institut is gratefully acknowledged. We thank Karen Korsholm for the illustration showing CAF23. We are grateful to Tillmann Hanns Pape and Klaus Qvortrup at the Core Facility for Integrated Microscopy, Faculty of Health and Medical Sciences, University of Copenhagen, for their support with the CryoTEM microscope. The project was funded by the Danish Research Council (Project ID: DFF - 1331-00068A), ADITEC ADITEC (EU grant number 280873), UNISEC (EU grant number 602012), and TBVAC (EU grant no 643381).

\section{REFERENCES}

(1) Czerkinsky, C.; Holmgren, J. Vaccines against Enteric Infections for the Developing World. Philos. Trans. R. Soc., B 2015, 370, 370.

(2) Dougan, G.; Huett, A.; Clare, S. Vaccines against Human Enteric Bacterial Pathogens. Br. Med. Bull. 2002, 62, 113-123.

(3) Mora, J. R.; Iwata, M.; Eksteen, B.; Song, S. Y.; Junt, T.; Senman, B.; Otipoby, K. L.; Yokota, A.; Takeuchi, H.; Ricciardi-Castagnoli, P.; Rajewsky, K.; Adams, D. H.; von Andrian, U. H. Generation of GutHoming Iga-Secreting B Cells by Intestinal Dendritic Cells. Science 2006, 314, 1157-1160.

(4) Hall, J. A.; Grainger, J. R.; Spencer, S. P.; Belkaid, Y. The Role of Retinoic Acid in Tolerance and Immunity. Immunity 2011, 35, 1322.

(5) Klebanoff, C. A.; Spencer, S. P.; Torabi-Parizi, P.; Grainger, J. R.; Roychoudhuri, R.; Ji, Y.; Sukumar, M.; Muranski, P.; Scott, C. D.; Hall, J. A.; Ferreyra, G. A.; Leonardi, A. J.; Borman, Z. A.; Wang, J.; Palmer, D. C.; Wilhelm, C.; Cai, R.; Sun, J.; Napoli, J. L.; Danner, R. L. Retinoic Acid Controls the Homeostasis of Pre-Cdc-Derived Splenic and Intestinal Dendritic Cells. J. Exp. Med. 2013, 210, 19611976.

(6) Pino-Lagos, K.; Guo, Y.; Brown, C.; Alexander, M. P.; Elgueta, R.; Bennett, K. A.; De Vries, V.; Nowak, E.; Blomhoff, R.; Sockanathan, S.; Chandraratna, R. A.; Dmitrovsky, E.; Noelle, R. J. A Retinoic Acid-Dependent Checkpoint in the Development of 
CD4+ T Cell-Mediated Immunity. J. Exp. Med. 2011, 208, 17671775.

(7) Iwata, M.; Hirakiyama, A.; Eshima, Y.; Kagechika, H.; Kato, C.; Song, S. Y. Retinoic Acid Imprints Gut-Homing Specificity on $\mathrm{T}$ Cells. Immunity 2004, 21, 527-538.

(8) Ross, A. C. Vitamin a and Retinoic Acid in T Cell-Related Immunity. Am. J. Clin. Nutr. 2012, 96, 1166S-1172S.

(9) Molenaar, R.; Greuter, M.; van der Marel, A. P.; Roozendaal, R.; Martin, S. F.; Edele, F.; Huehn, J.; Forster, R.; O’Toole, T.; Jansen, W.; Eestermans, I. L.; Kraal, G.; Mebius, R. E. Lymph Node Stromal Cells Support Dendritic Cell-Induced Gut-Homing of T Cells. J. Immunol. 2009, 183, 6395-6402.

(10) Hammerschmidt, S. I.; Friedrichsen, M.; Boelter, J.; Lyszkiewicz, M.; Kremmer, E.; Pabst, O.; Forster, R. Retinoic Acid Induces Homing of Protective $\mathrm{T}$ and $\mathrm{B}$ Cells to the Gut after Subcutaneous Immunization in Mice. J. Clin. Invest. 2011, 121, 30513061.

(11) Mwanza-Lisulo, M.; Kelly, P. Potential for Use of Retinoic Acid as an Oral Vaccine Adjuvant. Philos. Trans. R. Soc., B 2015, 370, 370.

(12) Sinha, R.; Howlader, D. R.; Ta, A.; Mitra, S.; Das, S.; Koley, H. Retinoic Acid Pre-Treatment Down Regulates V. Cholerae Outer Membrane Vesicles Induced Acute Inflammation and Enhances Mucosal Immunity. Vaccine 2017, 35, 3534-3547.

(13) Tan, X.; Sande, J. L.; Pufnock, J. S.; Blattman, J. N.; Greenberg, P. D. Retinoic Acid as a Vaccine Adjuvant Enhances Cd8+ T Cell Response and Mucosal Protection from Viral Challenge. J. virol. 2011, 85, 8316-8327.

(14) Yu, M.; Vajdy, M. A Novel Retinoic Acid, Catechin Hydrate and Mustard Oil-Based Emulsion for Enhanced Cytokine and Antibody Responses against Multiple Strains of Hiv-1 Following Mucosal and Systemic Vaccinations. Vaccine 2011, 29, 2429-2436.

(15) van Dissel, J. T.; Joosten, S. A.; Hoff, S. T.; Soonawala, D.; Prins, C.; Hokey, D. A.; O’Dee, D. M.; Graves, A.; ThierryCarstensen, B.; Andreasen, L. V.; Ruhwald, M.; de Visser, A. W.; Agger, E. M.; Ottenhoff, T. H.; Kromann, I.; Andersen, P. A Novel Liposomal Adjuvant System, Caf01, Promotes Long-Lived Mycobacterium Tuberculosis-Specific T-Cell Responses in Human. Vaccine 2014, 32, 7098-7107.

(16) Christensen, D.; Agger, E. M.; Andreasen, L. V.; Kirby, D.; Andersen, P.; Perrie, Y. Liposome-Based Cationic Adjuvant Formulations (Caf): Past, Present, and Future. J. Liposome Res. 2009, 19, 2-11.

(17) Davidsen, J.; Rosenkrands, I.; Christensen, D.; Vangala, A.; Kirby, D.; Perrie, Y.; Agger, E. M.; Andersen, P. Characterization of Cationic Liposomes Based on Dimethyldioctadecylammonium and Synthetic Cord Factor from M. Tuberculosis (Trehalose 6,6'Dibehenate)-a Novel Adjuvant Inducing Both Strong Cmi and Antibody Responses. Biochim. Biophys. Acta, Biomembr. 2005, 1718, $22-31$.

(18) Agger, E. M.; Rosenkrands, I.; Hansen, J.; Brahimi, K.; Vandahl, B. S.; Aagaard, C.; Werninghaus, K.; Kirschning, C.; Lang, R.; Christensen, D. Cationic Liposomes Formulated with Synthetic Mycobacterial Cordfactor (Caf01): A Versatile Adjuvant for Vaccines with Different Immunological Requirements. PLoS One 2008, 3, No. e3116.

(19) Olsen, A. W.; Theisen, M.; Christensen, D.; Follmann, F.; Andersen, P. Protection against Chlamydia Promoted by a Subunit Vaccine (Cth1) Compared with a Primary Intranasal Infection in a Mouse Genital Challenge Model. PLoS One 2010, 5, No. e10768.

(20) Rosenkrands, I.; Vingsbo-Lundberg, C.; Bundgaard, T. J.; Lindenstrom, T.; Enouf, V.; van der Werf, S.; Andersen, P.; Agger, E. M. Enhanced Humoral and Cell-Mediated Immune Responses after Immunization with Trivalent Influenza Vaccine Adjuvanted with Cationic Liposomes. Vaccine 2011, 29, 6283-6291.

(21) Karlsson, I.; Brandt, L.; Vinner, L.; Kromann, I.; Andreasen, L. V.; Andersen, P.; Gerstoft, J.; Kronborg, G.; Fomsgaard, A. Adjuvanted Hla-Supertype Restricted Subdominant Peptides Induce New T-Cell Immunity During Untreated Hiv-1-Infection. Clin. Immunol. 2013, 146, 120-130.
(22) Fomsgaard, A.; Karlsson, I.; Gram, G.; Schou, C.; Tang, S.; Bang, P.; Kromann, I.; Andersen, P.; Andreasen, L. V. Development and Preclinical Safety Evaluation of a New Therapeutic Hiv-1 Vaccine Based on 18 T-Cell Minimal Epitope Peptides Applying a Novel Cationic Adjuvant Caf01. Vaccine 2011, 29, 7067-7074.

(23) Roman, V. R. G.; Jensen, K. J.; Jensen, S. S.; Leo-Hansen, C.; Jespersen, S.; Te, D. D.; Rodrigues, C. M.; Janitzek, C. M.; Vinner, L.; Katzenstein, T. L.; Andersen, P.; Kromann, I.; Andreasen, L. V.; Karlsson, I.; Fomsgaard, A. Therapeutic Vaccination Using Cationic Liposome-Adjuvanted Hiv Type 1 Peptides Representing HlaSupertype-Restricted Subdominant T Cell Epitopes: Safety, Immunogenicity, and Feasibility in Guinea-Bissau. AIDS Res. Hum. Retroviruses 2013, 29, 1504-1512.

(24) Agger, E. M.; Rosenkrands, I.; Hansen, J.; Brahimi, K.; Vandahl, B. S.; Aagaard, C.; Werninghaus, K.; Kirschning, C.; Lang, R.; Christensen, D.; Theisen, M.; Follmann, F.; Andersen, P. Cationic Liposomes Formulated with Synthetic Mycobacterial Cordfactor (Caf01): A Versatile Adjuvant for Vaccines with Different Immunological Requirements. PLoS One 2008, 3, No. e3116.

(25) Nordly, P.; Korsholm, K. S.; Pedersen, E. A.; Khilji, T. S.; Franzyk, H.; Jorgensen, L.; Nielsen, H. M.; Agger, E. M.; Foged, C. Incorporation of a Synthetic Mycobacterial Monomycoloyl Glycerol Analogue Stabilizes Dimethyldioctadecylammonium Liposomes and Potentiates Their Adjuvant Effect in Vivo. Eur. J. Pharm. Biopharm. 2011, 77, 89-98.

(26) Bachmann, M. F.; Jennings, G. T. Vaccine Delivery: A Matter of Size, Geometry, Kinetics and Molecular Patterns. Nat. Rev. Immunol. 2010, 10, 787-796.

(27) Manolova, V.; Flace, A.; Bauer, M.; Schwarz, K.; Saudan, P.; Bachmann, M. F. Nanoparticles Target Distinct Dendritic Cell Populations According to Their Size. Eur. J. Immunol. 2008, 38, 1404-1413.

(28) Swartz, M. A. The Physiology of the Lymphatic System. Adv. Drug Delivery Rev. 2001, 50, 3-20.

(29) Ohtake, S.; Schebor, C.; Palecek, S. P.; de Pablo, J. J. Phase Behavior of Freeze-Dried Phospholipid-Cholesterol Mixtures Stabilized with Trehalose. Biochim. Biophys. Acta, Biomembr. 2005, 1713, $57-64$.

(30) Gregoriadis, G.; Davis, C. Stability of Liposomes in Vivo and in Vitro Is Promoted by Their Cholesterol Content and the Presence of Blood Cells. Biochem. Biophys. Res. Commun. 1979, 89, 1287-1293.

(31) Kaur, R.; Henriksen-Lacey, M.; Wilkhu, J.; Devitt, A.; Christensen, D.; Perrie, Y. Effect of Incorporating Cholesterol into Dda:Tdb Liposomal Adjuvants on Bilayer Properties, Biodistribution, and Immune Responses. Mol. Pharmaceutics 2014, 11, 197-207.

(32) Lee, S. C.; Lee, K. E.; Kim, J. J.; Lim, S. H. The Effect of Cholesterol in the Liposome Bilayer on the Stabilization of Incorporated Retinol. J. Liposome Res. 2005, 15, 157-166.

(33) Levine, M. M. Immunogenicity and Efficacy of Oral Vaccines in Developing Countries: Lessons from a Live Cholera Vaccine. BMC Biol. 2010, 8, 129.

(34) Madhi, S. A.; Cunliffe, N. A.; Steele, D.; Witte, D.; Kirsten, M.; Louw, C.; Ngwira, B.; Victor, J. C.; Gillard, P. H.; Cheuvart, B. B.; Han, H. H.; Neuzil, K. M. Effect of Human Rotavirus Vaccine on Severe Diarrhea in African Infants. N. Engl. J. Med. 2010, 362, 289298.

(35) Dietrich, J.; Andreasen, L. V.; Andersen, P.; Agger, E. M. Inducing Dose Sparing with Inactivated Polio Virus Formulated in Adjuvant Caf01. PLoS One 2014, 9, No. e100879.

(36) Evans, T. I.; Reeves, R. K. All-Trans-Retinoic Acid Imprints Expression of the Gut-Homing Marker Alpha 4 Beta 7 While Suppressing Lymph Node Homing of Dendritic Cells. Clin. Vaccine Immunol. 2013, 20, 1642-1646.

(37) Parthasarathy, R.; Gilbert, B.; Mehta, K. Aerosol Delivery of Liposomal All-Trans-Retinoic Acid to the Lungs. Cancer Chemother. Pharmacol. 1999, 43, 277-283.

(38) Watson, D. S.; Huang, Z.; Szoka, F. C., Jr. All-Trans Retinoic Acid Potentiates the Antibody Response in Mice to a Lipopeptide 
Antigen Adjuvanted with Liposomal Lipid A. Immunol. Cell Biol. 2009, 87, 630-633.

(39) Dos Santos, N.; Allen, C.; Doppen, A. M.; Anantha, M.; Cox, K. A.; Gallagher, R. C.; Karlsson, G.; Edwards, K.; Kenner, G.; Samuels, L.; Webb, M. S.; Bally, M. B. Influence of Poly(Ethylene Glycol) Grafting Density and Polymer Length on Liposomes: Relating Plasma Circulation Lifetimes to Protein Binding. Biochim. Biophys. Acta, Biomembr. 2007, 1768, 1367-1377.

(40) Ross, A. C.; Chen, Q.; Ma, Y. Augmentation of Antibody Responses by Retinoic Acid and Costimulatory Molecules. Semin. Immunol. 2009, 21, 42-50.

(41) Iwata, M.; Eshima, Y.; Kagechika, H. Retinoic Acids Exert Direct Effects on T Cells to Suppress Th1 Development and Enhance Th2 Development Via Retinoic Acid Receptors. Int. Immunol. 2003, 15, 1017-1025.

(42) Nozaki, Y.; Yamagata, T.; Yoo, B. S.; Sugiyama, M.; Ikoma, S.; Kinoshita, K.; Funauchi, M.; Kanamaru, A. The Beneficial Effects of Treatment with All-Trans-Retinoic Acid Plus Corticosteroid on Autoimmune Nephritis in Nzb/Wf Mice. Clin. Exp. Immunol. 2005, 139, 74-83.

(43) DeCicco, K. L.; Zolfaghari, R.; Li, N.; Ross, A. C. Retinoic Acid and Polyriboinosinic Acid Act Synergistically to Enhance the Antibody Response to Tetanus Toxoid During Vitamin a Deficiency: Possible Involvement of Interleukin-2 Receptor-Beta, Signal Transducer and Activator of Transcription-1, and Interferon Regulatory Factor-1. J. Infect. Dis. 2000, 182, 29-36.

(44) DeCicco, K. L.; Youngdahl, J. D.; Ross, A. C. All-TransRetinoic Acid and Polyriboinosinic: Polyribocytidylic Acid in Combination Potentiate Specific Antibody Production and CellMediated Immunity. Immunology 2001, 104, 341-348.

(45) Ma, Y.; Chen, Q.; Ross, A. C. Retinoic Acid and Polyriboinosinic:Polyribocytidylic Acid Stimulate Robust Anti-Tetanus Antibody Production While Differentially Regulating Type 1/ Type 2 Cytokines and Lymphocyte Populations. J. Immunol. 2005, 174, 7961-7969.

(46) Eriksen, A. B.; Torgersen, M. L.; Holm, K. L.; Abrahamsen, G.; Spurkland, A.; Moskaug, J. O.; Simonsen, A.; Blomhoff, H. K. Retinoic Acid-Induced Igg Production in Tlr-Activated Human Primary B Cells Involves Ulk1-Mediated Autophagy. Autophagy 2015, 11, 460-471.

(47) Ma, Y.; Ross, A. C. The Anti-Tetanus Immune Response of Neonatal Mice Is Augmented by Retinoic Acid Combined with Polyriboinosinic:Polyribocytidylic Acid. Proc. Natl. Acad. Sci. U. S. A. 2005, 102, 13556-13561.

(48) Schoenen, H.; Bodendorfer, B.; Hitchens, K.; Manzanero, S.; Werninghaus, K.; Nimmerjahn, F.; Agger, E. M.; Stenger, S.; Andersen, P.; Ruland, J.; Brown, G. D.; Wells, C.; Lang, R. Cutting Edge: Mincle Is Essential for Recognition and Adjuvanticity of the Mycobacterial Cord Factor and Its Synthetic Analog TrehaloseDibehenate. J. Immunol. 2010, 184, 2756-2760.

(49) Villablanca, E. J.; Wang, S.; de Calisto, J.; Gomes, D. C.; Kane, M. A.; Napoli, J. L.; Blaner, W. S.; Kagechika, H.; Blomhoff, R.; Rosemblatt, M.; Bono, M. R.; von Andrian, U. H.; Mora, J. R. Myd88 and Retinoic Acid Signaling Pathways Interact to Modulate Gastrointestinal Activities of Dendritic Cells. Gastroenterology 2011, $141,176-185$.

(50) Hammerschmidt, S. I.; Ahrendt, M.; Bode, U.; Wahl, B.; Kremmer, E.; Forster, R.; Pabst, O. Stromal Mesenteric Lymph Node Cells Are Essential for the Generation of Gut-Homing T Cells in Vivo. J. Exp. Med. 2008, 205, 2483-2490.

(51) Chen, X.; Esplin, B. L.; Garrett, K. P.; Welner, R. S.; Webb, C. F.; Kincade, P. W. Retinoids Accelerate B Lineage Lymphoid Differentiation. J. Immunol. 2008, 180, 138-145.

(52) Seo, G. Y.; Jang, Y. S.; Kim, H. A.; Lee, M. R.; Park, M. H.; Park, S. R.; Lee, J. M.; Choe, J.; Kim, P. H. Retinoic Acid, Acting as a Highly Specific Iga Isotype Switch Factor, Cooperates with Tgf-Beta 1 to Enhance the Overall Iga Response. J. Leukocyte Biol. 2013, 94, $325-335$.
(53) Ma, Y.; Ross, A. C. Toll-Like Receptor 3 Ligand and Retinoic Acid Enhance Germinal Center Formation and Increase the Tetanus Toxoid Vaccine Response. Clin. Vaccine. Immunol. 2009, 16, 14761484.

(54) Pantazi, E.; Marks, E.; Stolarczyk, E.; Lycke, N.; Noelle, R. J.; Elgueta, R. Cutting Edge: Retinoic Acid Signaling in B Cells Is Essential for Oral Immunization and Microflora Composition. J. Immunol. 2015, 195, 1368-1371.

(55) Evans, T. G.; Schrager, L.; Thole, J. Status of Vaccine Research and Development of Vaccines for Tuberculosis. Vaccine 2016, 34, 2911-2914.

(56) Olsen, A. W.; Lorenzen, E. K.; Follmann, F.; Andersen, P. A Polyprotein Vaccine Promotes Both Strong Th1 and Neutralizing Antibody Responses and Confers Efficient Protection against Genital C. trachomatis Infection in Mice and Pigs. Thirteenth International Symposium on Human Chlamydial Infections; Pacific Grove, CA, 2014. 